\title{
Total Coloring of Central Graphs of a Path, a Cycle and a Star
}

\author{
S. Sudha ${ }^{1}$, K. Manikandan ${ }^{2}$ \\ ${ }^{1}$ Professor, Ramanujan Institute for Advanced, Study in Mathematics, University of Madras, \\ Chennai, Tamil nadu, India \\ ${ }^{2}$ Assistant Professor, Department of Mathematics, Guru Nanak College, Chennai, Tamil nadu, India \\ *Corresponding Author: K. Manikandan, Assistant Professor, Department of Mathematics, Guru Nanak \\ College, Chennai, Tamil nadu, India
}

\begin{abstract}
The total chromatic number of a graph $G$ is defined to be the minimum number of colors needed to color the vertices and edges of a graph in such a way that no two adjacent vertices, no two adjacent edges and no incident vertex and edge are given the same color. In this paper, we discussed the total coloring and total chromatic number of the central graphs of a path, a cycle and a star.
\end{abstract}

Keywords: Central graph; Path; Cycle; Star; Total coloring; Total chromatic number.

\section{INTRODUCTION}

Bezhad [1] introduced the concept of total coloring and found the chromatic number of some simple graphs. If $G=(V(G), E(G))$ is a graph with the vertex set $V(G)$ and the edge set $E(G)$, a proper total coloring of $G$ is an assignment of colors to the vertices and the edges in such a way that

1. no two adjacent vertices are assigned with the same color,

2. no two adjacent edges are assigned with the same color and

3. no edge and its end vertices are assigned with the same color.

The total chromatic number of a graph $G$ is the minimum number of colors that required to produce a total coloring of and is denoted by $\chi_{t c}(G)$. Bezhad conjectured that for any graph of maximum degree $\Delta(G)$ has a total chromatic number satisfying the condition $\Delta(G)+1 \leq \chi_{t c}(G) \leq \Delta(G)+2$. This conjecture is known as the total coloring conjecture (TCC). This conjecture has been verified for many families of graphs and different graphs require different proofs depending on $\Delta(G)$. Bezhad et al. [2] have verified this conjecture for complete graphs and complete multipartite graphs. Rosenfeld [3] proved that the total chromatic number of every cubic graph is totally colorable with five colors. Borodin [4] proved this conjecture for planar graphs. Borodin et al. [5] proved that the chromatic number of a planar graph with maximum degree $\Delta(G) \geq 11$ is $\Delta(G)+1$. Yap [6] has given the total coloring of $r$-partite graphs and the graphs with degree $\Delta(G)=3, \Delta(G)=4$ and $\Delta(G) \geq|G|-5$. Seoud [7] has discussed about the total coloring of join of two paths, the cartesian product of two paths and the cartesian product of a path and a cycle. Hackmann et.al [8] discussed the circular total coloring of cubic circulant graphs. Sudha et.al $[9,10]$ have proved the total coloring and $(k, d)$-total coloring of prisms $Y_{n}$ and the total coloring for a prism graph of $n$-layers and a grid graph. Chen et al. [11] found that the total chromatic number of generalized Mycielski graphs. Vaidya et al. [12] proved that the total coloring of some cycle related graphs.

Definition 1.1. The central graph of a graph $G$ is obtained by subdividing each edge of $G$ exactly once and joining all the non-adjacent vertices of $G$.

The central graph of $G$ is denoted by $C(G)$.

In this paper, we have found the total chromatic number of the central graph of (i) a path, (ii) a cycle and (iii) a star. 


\section{CENTRAL GRAPH OF A PATH}

Consider a path $P_{5}$ with the vertex set $\left\{v_{1}, v_{2}, v_{3}, v_{4}, v_{5}\right\}$. If each edge of the path $P_{5}$ subdivided exactly once, let the new vertices be denoted by $u_{1}, u_{2}, u_{3}$ and $u_{4}$ as shown in "Fig. 1 ,".

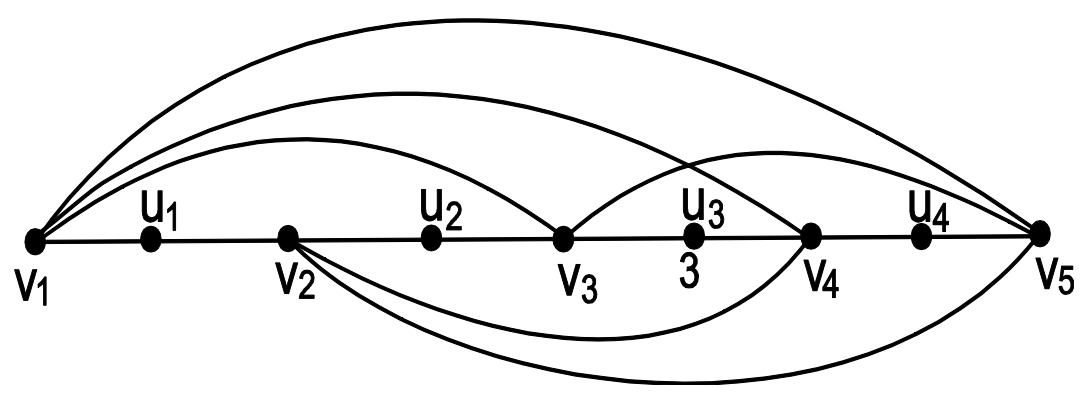

FIGURE 1. CENTRAL GRAPH OF PATH $P_{5}$

Now join the vertex $v_{1}$ with the vertices $v_{3}, v_{4}$ and $v_{5}$; the vertex $v_{2}$ with the vertices $v_{4}$ and $v_{5}$ and the vertex $v_{3}$ with the vertex $v_{5}$. The graph shown in "Fig. 1 ," is the central graph of the path $P_{5}$.

Theorem 2.1. The total chromatic number of the central graph of a path $P_{n}$ is given by $\chi_{t c}\left(C\left(P_{n}\right)\right)=$ (n, for odd $n$

$\{n+1$, for even $n$

Proof. Let the path $P_{n}$ has the vertex set $\left\{v_{i}, 1 \leq i \leq n\right\}$ and the edge set $\left\{v_{i} v_{i+1}, 1 \leq i \leq n-1\right\}$. As per the definition of the central graph of the path $P_{n}$, let the new vertices be $\left\{u_{i}, 1 \leq i \leq n-1\right\}$. The vertex set and the edge set of $C\left(P_{n}\right)$ are given by

$$
\begin{aligned}
& V\left(C\left(P_{n}\right)\right)=\left\{v_{i}, 1 \leq i \leq n\right\} \cup\left\{u_{i}, 1 \leq i \leq n-1\right\} \\
& E\left(C\left(P_{n}\right)\right)=\left\{\begin{array}{l}
\left\{v_{i} u_{i}, 1 \leq i \leq n-1\right\} \cup\left\{u_{i} v_{i+1}, 1 \leq i \leq n-1\right\} \\
\cup\left\{v_{i} v_{j}, 1 \leq i \leq n-2, i+2 \leq j \leq n\right\}
\end{array}\right\}
\end{aligned}
$$
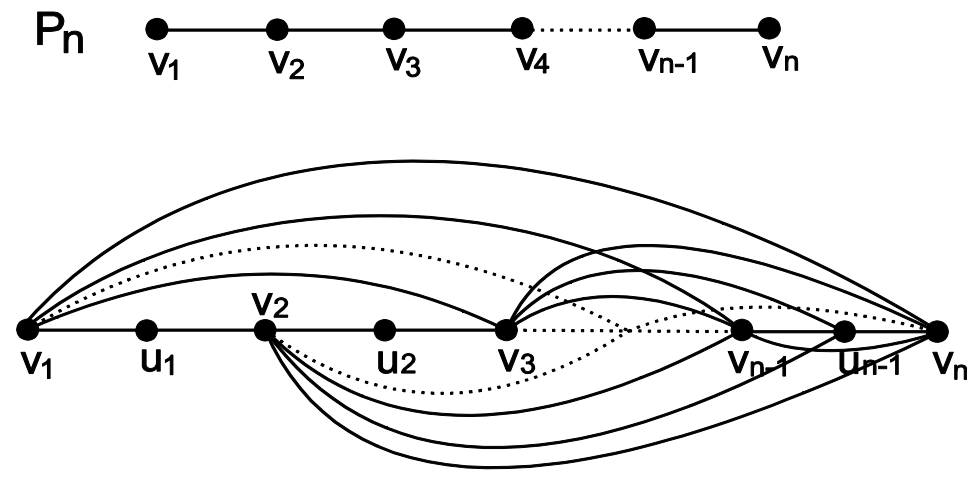

FIGURE 2. CENTRAL GRAPH OF PATH $P_{n}$

Define the functions $f_{1}$ and $f_{2}$ from the set of vertices and the set of edges to the set of colors for odd $n$ and even $n$ as follows:

Case 1: Let $n$ be odd.

For all $1 \leq i, j \leq n$,

$$
\begin{aligned}
f_{1}\left(v_{i}\right) & \equiv\left\{\begin{array}{l}
(2 i+1)(\bmod n), \text { if }(2 i+1) \not \equiv 0(\bmod n) \\
n, \text { otherwise }
\end{array}\right. \\
f_{1}\left(v_{n}\right) & =n \\
f_{1}\left(u_{i}\right) & \equiv\left\{\begin{array}{l}
2(i+1)(\bmod n), \text { if } 2(i+1) \not \equiv 0(\bmod n) \\
n, \text { otherwise }
\end{array}\right. \\
f_{1}\left(u_{n-1}\right) & =1 \\
f_{2}\left(v_{i} v_{j}\right) & \equiv\left\{\begin{array}{l}
(i+j)(\bmod n), \text { if }(i+j) \not \equiv 0(\bmod n), j>i+1 \\
n, \text { otherwise }
\end{array}\right.
\end{aligned}
$$




$$
f_{2}\left(v_{i} u_{j}\right) \equiv\left\{\begin{array}{l}
(i+j)(\bmod n), \text { if }(i+j) \not \equiv 0(\bmod n) \\
n, \text { otherwise }
\end{array}\right.
$$

With this type of coloring, the vertices and the edges of the central graph of the path $P_{n}$ for odd $n$ are properly total colored with $n$ colors.

Case 2: Let $n$ be even.

For all $1 \leq i, j \leq n$,

$$
\begin{aligned}
f_{1}\left(v_{i}\right) & \equiv\left\{\begin{array}{l}
(2 i+1)(\bmod (n+1)), \text { if }(2 i+1) \not \equiv 0(\bmod (n+1)) \\
n+1, \text { otherwise }
\end{array}\right. \\
f_{1}\left(u_{i}\right) & \equiv\left\{\begin{array}{l}
2(i+1)(\bmod (n+1)), \text { if } 2(i+1) \not \equiv 0(\bmod (n+1)) \\
n+1, \text { otherwise }
\end{array}\right. \\
f_{2}\left(v_{i} v_{j}\right) & \equiv\left\{\begin{array}{l}
(i+j)(\bmod (n+1)), \text { if }(i+j) \not \equiv 0(\bmod (n+1)), j>i+1 \\
n+1, \text { otherwise }
\end{array}\right. \\
f_{2}\left(v_{i} u_{j}\right) & \equiv\left\{\begin{array}{l}
(i+j)(\bmod (n+1)), \text { if }(i+j) \not \equiv 0(\bmod (n+1)) \\
n+1, \text { otherwise }
\end{array}\right.
\end{aligned}
$$

With this type of coloring, the vertices and the edges of the central graph of the path $P_{n}$ for even $n$ are properly total colored with $n+1$ colors.

Hence the total chromatic number of the central graph of the path $P_{n}$ is $n$ for odd $n$ and $n+1$ for even $n$.

Illustration 2.2. Consider the central graph of a path $P_{5}$.

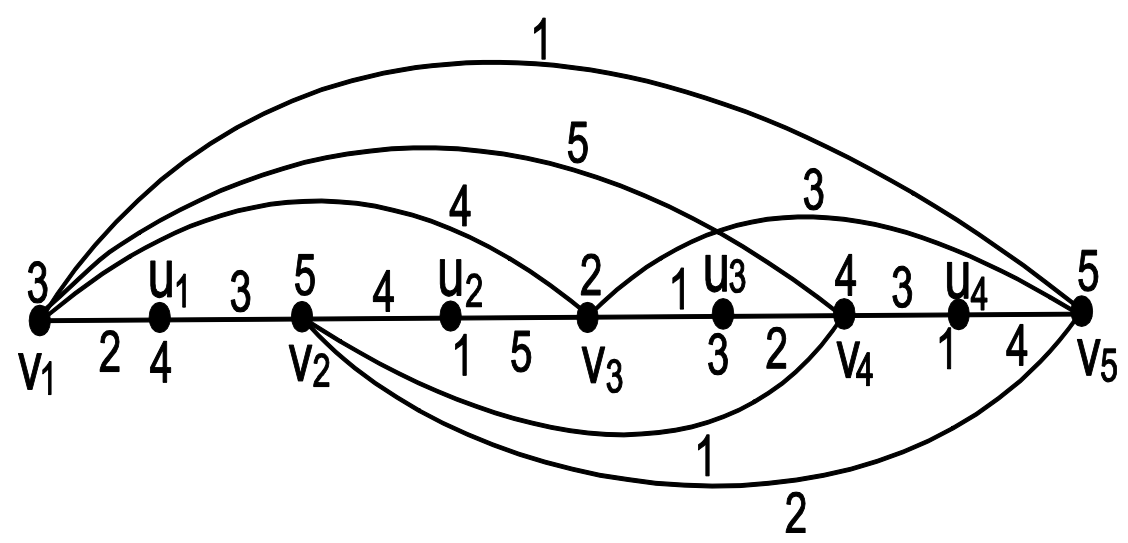

FIGURE 3. CENTRAL GRAPH OF PATH $P_{5}$

By using the coloring pattern as given in case 1 of theorem 2.1, the colors 1,2,3,4,5 to the vertices and the edges are assigned with the colors as shown in "Fig 3,".

The total chromatic number of the central graph of the path $P_{5}$ is 5 .

Illustration 2.3. Consider the central graph of a path $P_{6}$.

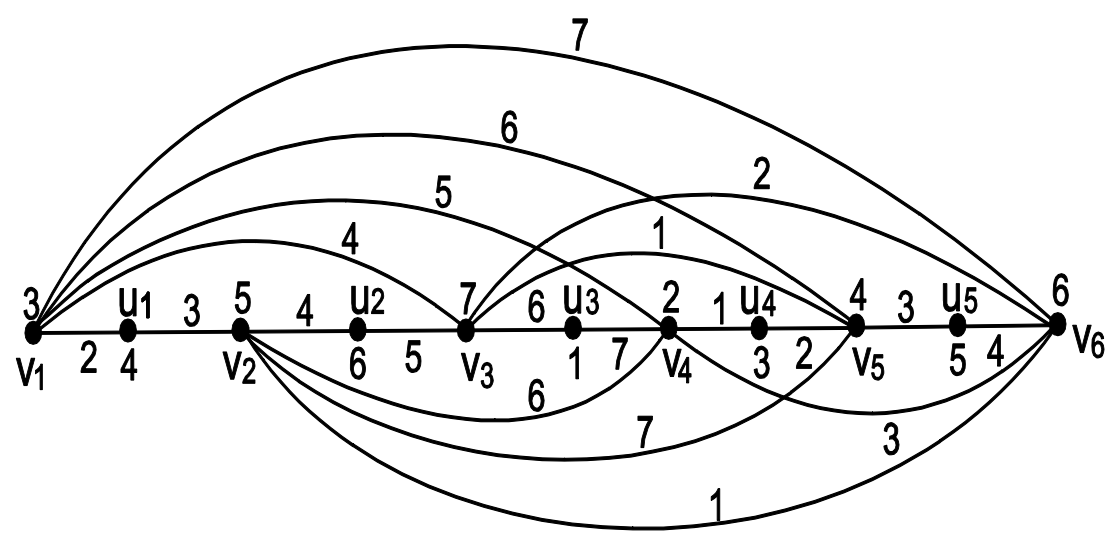

FIGURE 4. CENTRAL GRAPH OF PATH $P_{6}$ 
By using the coloring pattern as given in case 2 of theorem 2.1, the colors 1,2,3,4,5,6,7 to the vertices and the edges are assigned with the colors as shown in "Fig 3,".

The total chromatic number of the central graph of the path $P_{6}$ is 7 .

\section{CENTRAL GRAPH OF A CYCLE}

Consider a cycle $C_{5}$ with the vertex set $\left\{v_{1}, v_{2}, v_{3}, v_{4}, v_{5}\right\}$. If each edge of the cycle $C_{5}$ subdivided exactly once, let the new vertices be denoted by $u_{1}, u_{2}, u_{3}, u_{4}$ and $u_{5}$ as shown in "Fig. 5,".

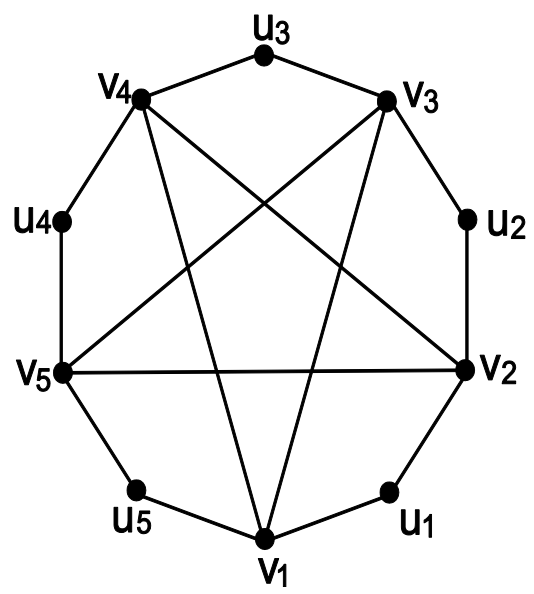

FIGURE 5. CENTRAL GRAPH OF CYCLE $C_{5}$

Now join the vertex $v_{1}$ with the vertices $v_{3}, v_{4}$ and $v_{5}$; the vertex $v_{2}$ with the vertices $v_{4}$ and $v_{5}$ and the vertex $v_{3}$ with the vertex $v_{5}$. The graph shown in "Fig. 5 ," is the central graph of the cycle $C_{5}$.

Theorem 3.1. The total chromatic number of the central graph of a cycle $C_{n}$ is given by $\chi_{t c}\left(C\left(C_{n}\right)\right)= \begin{cases}n, & \text { for odd } n \\ n+1, & \text { for even } n\end{cases}$

Proof. Let the cycle $C_{n}$ has the vertex set $\left\{v_{i}, 1 \leq i \leq n\right\}$ and the edge set $\left\{v_{i} v_{i+1}, 1 \leq i \leq n-1\right\} \cup$ $\left\{v_{n} v_{1}\right\}$. As per the definition of the central graph of the cycle $C_{n}$, let the new vertices be $\left\{u_{i}, 1 \leq i \leq\right.$ $n$ \}. The vertex set and the edge set of $C\left(C_{n}\right)$ are given by

$$
\begin{aligned}
& V\left(C\left(C_{n}\right)\right)=\left\{v_{i}, 1 \leq i \leq n\right\} \cup\left\{u_{i}, 1 \leq i \leq n-1\right\} \\
& E\left(C\left(C_{n}\right)\right)=\left\{\begin{array}{l}
\left\{v_{i} u_{i}, 1 \leq i \leq n-1\right\} \cup\left\{u_{i} v_{i+1}, 1 \leq i \leq n-1\right\} \\
\cup\left\{u_{n} v_{1}\right\}\left\{v_{i} v_{j}, 1 \leq i \leq n-2, i+2 \leq j \leq n\right\}
\end{array}\right\}
\end{aligned}
$$

Define the functions $f_{1}$ and $f_{2}$ from the set of vertices and the set of edges to the set of colors for odd $n$ and even $n$ as follows:

Case 1: Let $n$ be odd.

For all $1 \leq i, j \leq n$,

$$
\begin{aligned}
f_{1}\left(v_{i}\right) & \equiv\left\{\begin{array}{l}
(2 i+1)(\bmod n), \text { if }(2 i+1) \not \equiv 0(\bmod n) \\
n, \text { otherwise }
\end{array}\right. \\
f_{1}\left(u_{i}\right) & \equiv\left\{\begin{array}{l}
2(i+1)(\bmod n), \text { if } 2(i+1) \not \equiv 0(\bmod n) \\
n, \text { otherwise }
\end{array}\right. \\
f_{2}\left(v_{i} v_{j}\right) & \equiv\left\{\begin{array}{l}
(i+j)(\bmod n), \text { if }(i+j) \not \equiv 0(\bmod n), j>i+1 \\
n, \text { otherwise }
\end{array}\right. \\
f_{2}\left(v_{i} u_{j}\right) & \equiv\left\{\begin{array}{l}
(i+j)(\bmod n), \text { if }(i+j) \not \equiv 0(\bmod n) \\
n, \text { otherwise }
\end{array}\right.
\end{aligned}
$$

The above coloring satisfies the condition for a total coloring of the central graph of the cycle $C_{n}$ for odd $n$. Hence the total chromatic number of the central graph of the cycle $C_{n}$ is $n$ for odd $n$.

Case 2: Let $n$ be even.

For all $1 \leq i, j \leq n$, 


$$
\begin{aligned}
f_{1}\left(v_{i}\right) & \equiv\left\{\begin{array}{l}
(2 i+1)(\bmod (n+1)), \text { if }(2 i+1) \not \equiv 0(\bmod (n+1)) \\
n+1, \text { otherwise }
\end{array}\right. \\
f_{1}\left(u_{i}\right) & \equiv\left\{\begin{array}{l}
2(i+1)(\bmod (n+1)), \text { if } 2(i+1) \not \equiv 0(\bmod (n+1)) \\
n+1, \text { otherwise }
\end{array}\right. \\
f_{1}\left(u_{n}\right) & =1 \\
f_{2}\left(v_{i} v_{j}\right) & \equiv\left\{\begin{array}{l}
(i+j)(\bmod (n+1)), \text { if }(i+j) \not \equiv 0(\bmod (n+1)), j>i+1 \\
n+1, \text { otherwise }
\end{array}\right. \\
f_{2}\left(v_{i} u_{j}\right) & \equiv\left\{\begin{array}{l}
(i+j)(\bmod (n+1)), \text { if }(i+j) \not \equiv 0(\bmod (n+1)) \\
n+1, \text { otherwise }
\end{array}\right.
\end{aligned}
$$

The above coloring satisfies the condition for a total coloring of the central graph of the cycle $C_{n}$ for even $n$. Hence the total chromatic number of the central graph of the cycle $C_{n}$ is $n+1$ for even $n$.

Illustration 3.1. Consider the central graph of a cycle $C_{5}$.

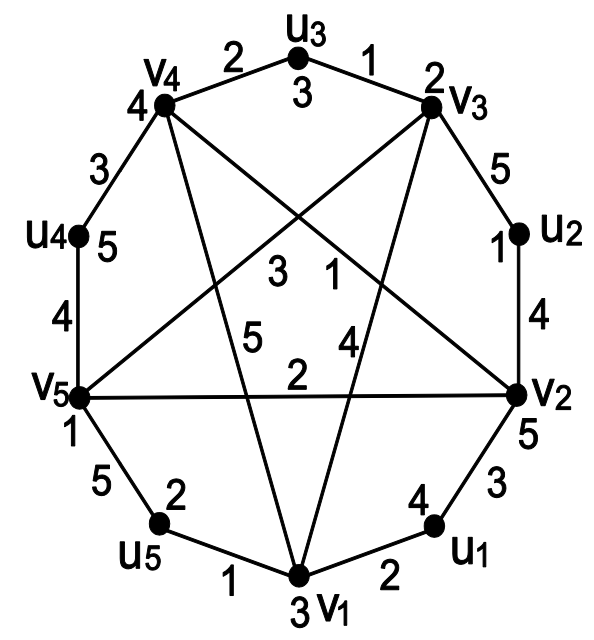

FIGURE 6. CENTRAL GRAPH OF A CYCLE $C_{5}$

By using the coloring pattern as given in case 1 of theorem 3.1, the colors 1,2,3,4,5 are assigned to the vertices and the edges are colored as shown in "Fig. 6,".

The total chromatic number of the central graph of the cycle $C_{5}$ is 5 .

Illustration 3.1. Consider the central graph of a cycle $C_{8}$.

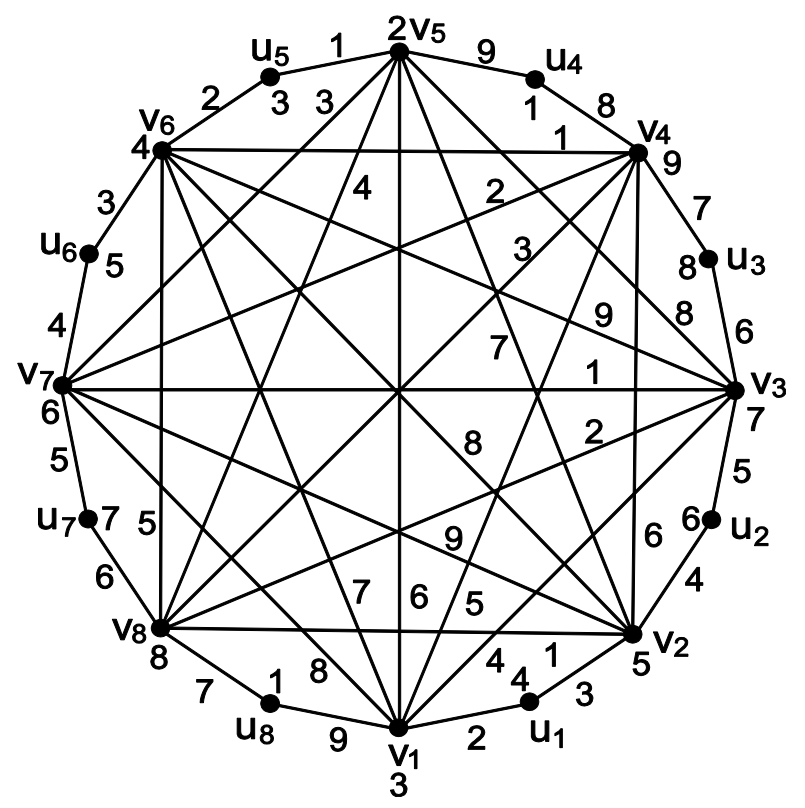

FIGURE 7. CENTRAL GRAPH OF A CYCLE $C_{8}$ 
By using the coloring pattern as given in case 2 of theorem 3.1 , the colors $1,2,3,4,5,6,7,8,9$ are assigned to the vertices and the edges are colored as shown in "Fig. 7,".

The total chromatic number of the central graph of the cycle $C_{8}$ is 9 .

\section{CENTRAL GRAPH OF A STAR}

Consider a star $K_{1,4}$ with the vertex set $\left\{v_{0}, v_{1}, v_{2}, v_{3}, v_{4}\right\}$. If each edge of the star $K_{1,4}$ is supersubdivided by the biparitite graph $K_{2,1}$, let the new vertices be denoted by $u_{1}, u_{2}, u_{3}$ and $u_{4}$ as shown in "Fig. 8,".

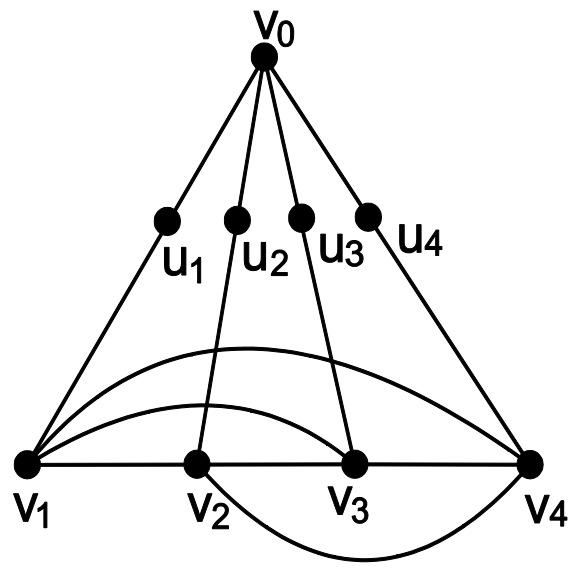

FIGURE 8. CENTRAL GRAPH OF A STAR $K_{1,4}$

No join the vertex $v_{1}$ with the vertices $v_{2}, v_{3}$ and $v_{4}$; the vertex $v_{2}$ with the vertices $v_{3}$ and $v_{4}$ and the vertex $v_{3}$ with the vertex $v_{4}$. The graph shown in "Fig. 8," is the central graph of the star $K_{1,4}$.

Theorem 4.1. The total chromatic number of the central graph of a star $K_{1, n}$ is $n+1$ for $n \geq 3$.

\section{Proof.}
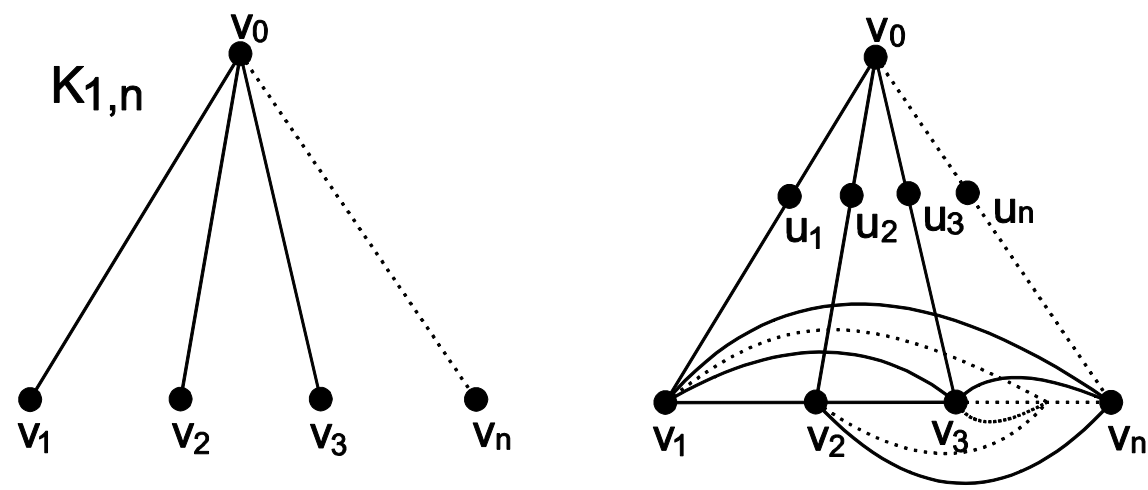

FIGURE 9. CENTRAL GRAPH OF A STAR $K_{1, n}$

Let $v_{0}$ be the central vertex and $v_{1}, v_{2}, v_{3}, \ldots, v_{n}$ be the pendent vertices of $K_{1,4}$. As per the definition of the central graph, let the new vertices be $u_{i}, 1 \leq i \leq n$. The vertex set and the edge set of $C\left(K_{1, n}\right)$ are given by

$$
\begin{array}{r}
V\left(C\left(K_{1, n}\right)\right)=\left\{v_{i}, 1 \leq i \leq n\right\} \cup\left\{u_{i}, 1 \leq i \leq n\right\} \cup\left\{v_{0}\right\} \\
\text { and } E\left(C\left(K_{1, n}\right)\right)=\left\{\begin{array}{l}
\left\{v_{0} u_{i}, 1 \leq i \leq n\right\} \cup\left\{u_{i} v_{i}, 1 \leq i \leq n\right\} \\
\cup\left\{v_{i} v_{j}, 1 \leq i \leq n-1, i+1 \leq j \leq n\right\}
\end{array}\right\}
\end{array}
$$

Define the function $f_{1}$ and $f_{2}$ from the set of vertices and the set of edges to the set of colors $\{1,2,3, \ldots, n\}$ as follows:

$$
\begin{aligned}
& f_{1}\left(v_{0}\right)=n+1, \\
& f_{1}\left(v_{i}\right)=i, 1 \leq i \leq n, \\
& f_{1}\left(u_{1}\right)=3 \text { and } f_{1}\left(u_{n}\right)=1
\end{aligned}
$$




$$
\begin{aligned}
f_{1}\left(u_{i}\right) & =i-1,2 \leq i \leq n-1, \\
f_{2}\left(v_{0} u_{i}\right) & =i, 1 \leq i \leq n, \\
f_{2}\left(v_{i} u_{i}\right) & =\left\{\begin{array}{l}
2 i(\bmod (n+1)), \text { if } 2 i \not \equiv 0(\bmod (n+1)), \\
n+1, \text { otherwise }
\end{array}\right. \\
f_{2}\left(v_{i} u_{i}\right) & =\left\{\begin{array}{l}
(i+j)(\bmod (n+1)), \text { if }(i+j) \not \equiv 0(\bmod (n+1)), j \geq i \\
n+1, \text { otherwise }
\end{array}\right.
\end{aligned}
$$

$$
\text { for } 1 \leq i, j \leq n \text {. }
$$

The above coloring satisfies the condition for a total coloring of the central graph of the star $K_{1, n}$. Hence the total chromatic number of the central graph of the star $K_{1, n}$ is $n+1$ for $n \geq 3$.

Illustration 4.1. Consider the central graph of a star $K_{1,6}$.

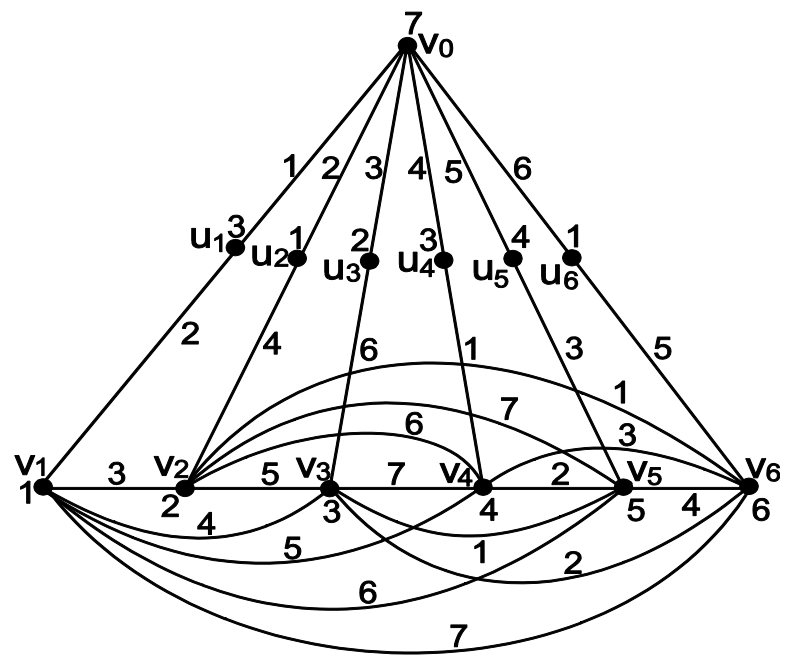

FIGURE 10. CENTRAL GRAPH OF A STAR $K_{1,6}$

By using the coloring pattern given in theorem 4.1, the colors 1,2,3,4,5,6,7 are assigned to the vertices and the edges with the colors as shown in "Fig. 10,"

\section{CONCLUSION}

The total coloring of the central graph of a path, a cycle and star are discussed in this paper and found the total chromatic numbers to be

$$
\begin{aligned}
& \text { 1. } \chi_{t c}\left(C\left(P_{n}\right)\right)= \begin{cases}n, & \text { for odd } n \\
n+1, & \text { for even } n\end{cases} \\
& \text { 2. } \chi_{t c}\left(C\left(C_{n}\right)\right)= \begin{cases}n, & \text { for odd } n \\
n+1, & \text { for even } n\end{cases} \\
& \text { 3. } \chi_{t c}\left(C\left(K_{1, n}\right)\right)=n+1 .
\end{aligned}
$$

\section{ACKNOWLEDGEMENTS}

The author thanks the anonymous referees for their useful comments and suggestions, which have improved the paper.

\section{REFERENCES}

[1] M. Behzad, "Graphs and their chromatic numbers," Ph.D. Thesis, Michigan state university, East Lansing, (1965).

[2] M. Behzad, G. Chartrand and J.K. Cooper, "The color numbers of complete graphs," Journal London Math.Soc., 42, 226-228, (1967).

[3] M. Rosenfeld, “On total colouring of certain graphs,” Israel J. Math. 9(3),396-402, 1971.

[4] O. V. Borodin, A. V. Kostochka and D. R. Woodall, "Total colouring of planar graphs with large maximum degree," J. Graph Theory, 26, 53 - 59, 1997. 
[5] O. V. Borodin, "On the total colouring of planar graphs," J. Reine Angew. Math., 394, 180 - 185, (1989).

[6] H. P. Yap, "Total colourings of graphs," Lecture Notes in Mathematics, 1623, Springer - Verlag, Berlin, (1996).

[7] M.A. Seoud, "Total chromatic numbers," Appl. Math. Lett. 5(6), pp. 37-39, (1992).

[8] A. Hackmann and A. Kemnitz, "Circular total coloring of cubic circulant graphs," J. Combin. Math. Combin. Cumput. 49, pp. 65-72, (2004).

[9] S. Sudha and K. Manikandan, "Total coloring and (k,d)-total coloring of prisms," Mathematical Sciences International Research Journal, 2(1), 11-13, (2014).

[10] S. Sudha and K. Manikandan, "General pattern of total coloring of a prism graph of n-layers and a grid graph," International Journal of innovative science and Modern Engineering, 3(3), pp 33-37, (2014).

[11] M. Chen, Xiaofeng, H. Li, L. Zhang, "Total chromatic number of generalized Mycielskian graphs," Discrete Math.334, 48-51, (2014).

[12] S.K. Vaidya, R. V.Isaac, "Total coloring of some cycle related graphs," ISOR Journal of Mathematics, 11(3), (2015).

\section{AUTHORS' BIOGRAPHY}

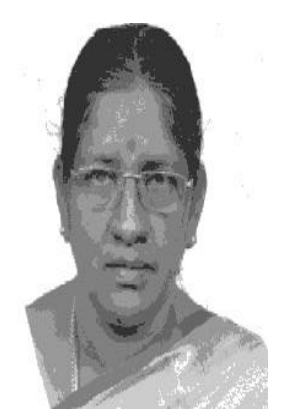

Dr. S. Sudha has got her Ph.D., in 1984. She has got 35 years of teaching and research experience. She is currently working as a Professor in Mathematics at the Ramanujan Institute for Advanced Study in Mathematics, University of Madras, Chennai - 600005. Her fields of interest are Computational Fluid Dynamics, Graph Theory, Fuzzy Graphs and Queueing Theory. She has published more than 25 articles in journals. She has also published some books.

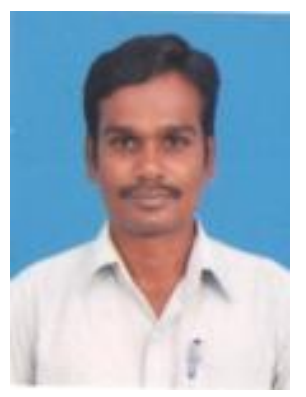

Dr. K. Manikandan has got him Ph.D., in 2017 at_Ramanujan Institute for Advanced Study in Mathematics, University of Madras, Chennai-600005. He has published six articles in a journals.

Citation: S. Sudha, K. Manikandan, "Total Coloring of Central Graphs of a Path, a Cycle and a Star ", International Journal of Scientific and Innovative Mathematical Research, vol. 5, no. 10, p. 15-22, 2017., http://dx.doi.org/10.20431/2347-3142.0510003

Copyright: () 2017 Authors. This is an open-access article distributed under the terms of the Creative Commons Attribution License, which permits unrestricted use, distribution, and reproduction in any medium, provided the original author and source are credited. 